\title{
Patientenverfügung als ökonomischer Faktor
}

\begin{abstract}
Nach einer Untersuchung aus den USA entstehen bei Patienten, die eine Patientenverfügung haben, geringere Kosten für die medizinische Versorgung in den letzten sechs Lebensmonaten. Diese Patienten sterben seltener im Krankenhaus und beanspruchen in größerem Ausmaß eine palliativmedizinische Versorgung. Das alles trifft aber nur für diejenigen Regionen zu, die beim regionalen Vergleich besonders viel Geld für die Medizin in der letzten Lebensphase ausgeben.
\end{abstract}

- Die Daten der prospektiven amerikanischen Health and Retirement Study und des nationalen Sterberegisters von 3302 Anspruchsberechtigten von Medicare, die zwischen 1998 und 2007 verstorben sind, wurden verlinkt und in einem multivariaten Regressionsmodell wurden Assoziationen zwischen dem Vorhanden- sein von Patientenverfügungen und den Ausgaben für die medizinische Versorgung in den letzten sechs Lebensmonaten in Relation zu den Gesamtausgaben von Medicare in der Herkunftsregion des Verstorbenen errechnet. Zusätzlich stellte man fest, wie viele Patienten im Krankenhaus und wie viele in anderen Einrichtungen verstorben waren.

Dabei ergab sich ein höchst differenziertes Bild. Das Vorhandensein einer Patientenverfügung führte pro Verstorbenem zu Minderausgaben von im Median 5585 \$, allerdings nur in Regionen, die beim Benchmarking überdurchschnittlich viel für die medizinische Versorgung in den letzten Lebensmonaten ausgaben. In Regionen mit nur niedrigen oder mittleren Ausgaben hatten Patientenverfügungen dagegen keinen Einfluss auf die Versorgungskosten.

Die Wahrscheinlichkeit, im Krankenhaus zu versterben, lag bei den Pati- enten mit Patientenverfügung in den Regionen mit hohen Ausgaben um 9,8\%, in denen mit nur geringen Ausgaben nur um 5,3\% niedriger als in der Vergleichsgruppe ohne Patientenverfügung. Ähnlich verhielt es sich mit der Betreuung in einem Hospiz. Personen mit Patientenverfügungen wurden in Regionen mit hohen Ausgaben mit 17\% höherer Wahrscheinlichkeit in einem Hospiz behandelt, in Regionen mit mittelhohen Ausgaben mit 11\%. Dagegen hatte die Patientenverfügung in Regionen mit niedrigen medizinischen Kosten keinen Einfluss auf die Wahrscheinlichkeit einer Hospizbetreuung.

\footnotetext{
- L. H. Nicholas et al.

Regional variation in the association between advance directives and end-of-life medicare expenditures. JAMA 306 (2011) 13, 1447-1453.
}

\section{Kommentar}

Die Interpretation dieser Untersuchung ist nicht leicht und lässt Raum für eine Fülle von Spekulationen. Ohne detaillierte Kenntnis der tatsächlichen Praxis in den jeweiligen Krankenhäusern sind die wahren Gründe für diese Ergebnisse wohl kaum auszumachen. So könnte es zum Beispiel sein, dass Patientenverfügungen gerade Krankenhäusern mit einer besonders aggressiven und damit kostspieligen Vorgehensweise beim Einsatz lebensverlängernder Maßnahmen in den Arm fallen und auf diese Weise weniger Kosten entstehen. In Regionen mit niedrigeren Ausgaben hat man diese Politik möglicherweise unabhängig vom Vorliegen einer Patientenverfügung bereits schon früher verfolgt.

Immerhin ist bemerkenswert, dass sich die Gesundheitsökonomen nun auch der Palliativmedizin angenommen haben. Es konnte nicht ausbleiben. Wie sagten englische Satiriker schon lange vor Karsten Vilmar: „Death is a great way of cutting down expenses."

H. S. FÜESSL =

Kostenbremse Patientenverfügung?

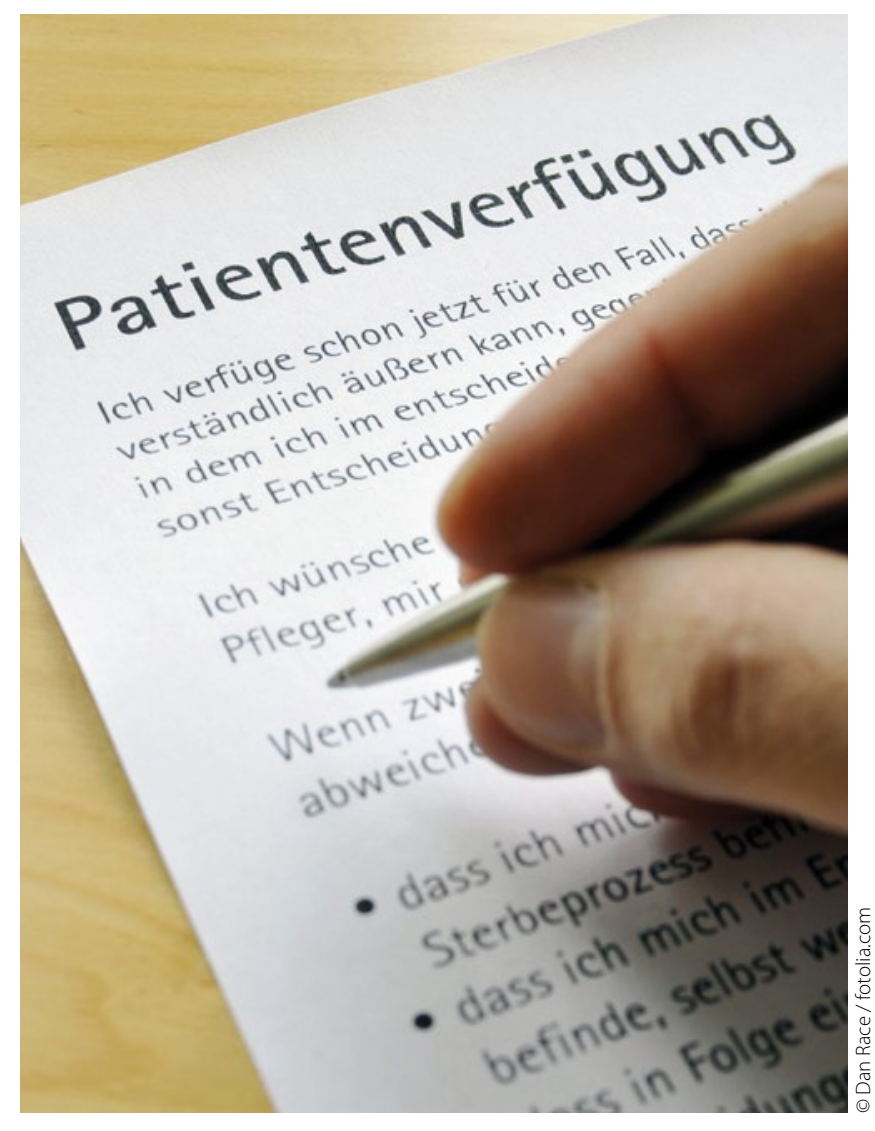

